\title{
BMJ Open Geographical and population disparities in timely access to prehospital and advanced level emergency care in New Zealand: a cross-sectional study
}

\author{
Rebbecca Lilley, ${ }^{\oplus 1}$ Brandon de Graaf, ${ }^{1}$ Bridget Kool, ${ }^{2}$ Gabrielle Davie, ${ }^{\oplus 1}$ \\ Papaarangi Reid, ${ }^{3}$ Bridget Dicker, ${ }^{4,5}$ lan Civil, ${ }^{6,7}$ Shanthi Ameratunga, ${ }^{2}$ \\ Charles Branas ${ }^{8}$
}

To cite: Lilley R, de Graaf B, Kool B, et al. Geographical and population disparities in timely access to prehospital and advanced level emergency care in New Zealand: a crosssectional study. BMJ Open 2019;9:e026026. doi:10.1136/ bmjopen-2018-026026

- Prepublication history for this paper is available online. To view these files, please visit the journal online (http://dx.doi org/10.1136/bmjopen-2018026026).

Received 14 August 2018 Revised 20 June 2019 Accepted 04 July 2019
Check for updates

(C) Author(s) (or their employer(s)) 2019. Re-use permitted under CC BY-NC. No commercial re-use. See rights and permissions. Published by BMJ.

For numbered affiliations see end of article.

Correspondence to Dr Rebbecca Lilley; rebbecca.lilley@ipru.otago.ac.nz

\section{ABSTRACT}

Objective Rapid access to advanced emergency medical and trauma care has been shown to significantly reduce mortality and disability. This study aims to systematically examine geographical access to prehospital care provided by emergency medical services (EMS) and advancedlevel hospital care, for the smallest geographical units used in New Zealand and explores national disparities in geographical access to these services.

Design Observational study involving geospatial analysis estimating population access to EMS and advanced-level hospital care.

Setting Population access to advanced-level hospital care via road and air EMS across New Zealand.

Participants New Zealand population usually resident within geographical census meshblocks.

Primary and secondary outcome measures The proportion of the resident population with calculated EMS access to advanced-level hospital care within 60 min was examined by age, sex, ethnicity, level of deprivation and population density to identify disparities in geographical access.

Results An estimated $16 \%$ of the New Zealand population does not have timely EMS access to advanced-level hospital care via road or air. The 700000 New Zealanders without timely access lived mostly in areas of lowmoderate population density. Indigenous Māori, New Zealand European and older New Zealanders were less likely to have timely access.

Conclusions These findings suggest that in New Zealand, geographically marginalised groups which tend to be rural and remote communities with disproportionately more indigenous Māori and older adults have poorer EMS access to advanced-level hospitals. Addressing these inequities in rapid access to medical care may lead to improvements in survival that have been documented for people who experience medical or surgical emergencies.

\section{INTRODUCTION}

Globally, millions of people with life-threatening injuries or health events require timely access to advanced healthcare services, including prehospital emergency services, to prevent needless mortality and morbidity. ${ }^{1}$
Strengths and limitations of this study

- This study is the first step in assessing access to emergency medical services (EMS) in New Zealand, a long, narrow and geographically challenging island nation in the South Pacific with a small, geographically dispersed population.

- This study expands on previous international assessments of timely access to emergency hospital-level care by mapping exact locations of EMS response.

- The choice of geospatial software and consciously conservative assumptions made are likely to underestimate the impact the access estimates presented.

- The EMS scenario modelled in our analysis fits a theoretical 'scoop \& run' strategy to prehospital care, and does not consider other possible prehospital strategies, such as a differentiated strategy, or rapid delivery of specialist medical expertise to the patient.

The public health burden of time-critical medical emergencies can be further exacerbated in countries with challenging terrain, long travel distances and dispersed populations, despite relative economic standing and available resources. ${ }^{2}$ New Zealand is one country where these natural and population features are a daily challenge to the timely and equitable delivery of prehospital emergency care.

Death and disability due to acute medical emergencies can be significantly reduced by the timely provision of prehospital emergency healthcare, thereby presenting many opportunities for tertiary prevention. Optimal models of trauma care delivery, focusing on a continuum of timely emergency medical services (EMS) to efficient in-hospital care, start with the rapid provision of prehospital EMS and provide the best chance for survival and rehabilitation following injury. ${ }^{1} 34 \mathrm{~A}$ 
time sensitive approach has also been proven to benefit patients requiring EMS following cardiac, stroke and vascular events. ${ }^{56}$

EMS are a vital entry point into the continuum of acute emergency healthcare, as their primary role is to rapidly meet the emergency prehospital care needs of patients following time-critical injury or health events. In terms of receiving hospital-level treatment, the first hour, commonly referred to as the 'golden hour', is generally considered to be the most critical in terms of receiving definitive treatment for cases with time-critical injuries or health conditions. ${ }^{4}$ Although not supported by a strong evidence-base, access to EMS and advanced-level hospital care is often judged against this threshold. ${ }^{78}$ Disparities in timely access to advanced-level hospitals have been documented in numerous countries, identifying higher risk of delayed access for communities and vulnerable groups. ${ }^{79-12}$

New Zealand's mountainous terrain, long travel distances and relatively small and geographically dispersed population of 4.2 million can severely hinder physical/transport access to advanced hospital-level care impacting timely and equitable delivery of prehospital EMS. New Zealand's population density is relatively low at 15 people $/ \mathrm{km}^{2}$, considerably less than countries with similar land area such as the UK with 243 people/ $\mathrm{km}^{2}$. Despite having low overall population density, New Zealand is highly urbanised, with $86 \%$ of the population living in urban areas. ${ }^{13}$ Case reviews in New Zealand suggest there are opportunities to improve prehospital EMS and reduce the number of prehospital deaths. ${ }^{1415}$

This study systematically examines geographical proximity and access to prehospital care provided by EMS and advanced-level hospital care, for the smallest geographical units used in New Zealand, in order to identify opportunities to improve survivability and reduce disability following time-critical medical emergencies, and explores national disparities in geographical access to these services.

\section{METHODS}

\section{Advanced level hospital care in New Zealand}

There are three levels of classification for New Zealand hospitals, relating to their capability to provide the appropriate services for seriously injured or ill patients (table 1).
New Zealand has a unique system where by trauma response, treatment and rehabilitation are funded by the universal, no-fault, publicly funded Accident Compensation Corporation (ACC). In contrast, medical response, treatment and rehabilitation is publicly funded by each individual district health board with some part-charges for ambulance services.

\section{Data sources}

For this study, we used census 2013 (most recent publicly available data) meshblock boundary and characteristics information available from Statistics New Zealand. ${ }^{16}$ Meshblocks are the smallest geographical unit available. In the 2013 census, there were 45989 meshblocks ranging in size from part of a city block containing approximately 110 people to a large area of rural land containing approximately 60 people. In addition to the boundary shapefiles, we obtained sociodemographic, deprivation and urbanity characteristics of the population usually residing within each meshblock. ${ }^{16}$

The physical addresses of advanced-level (levels 1 and 2) hospitals were converted to geographical longitude-latitude coordinates. The locations of road ambulance stations as at 2014/2015 were obtained from the two providers of these services in New Zealand, St. John Ambulance (New Zealand's major provider serving 98\% of the population) and Wellington Free Ambulance. Air EMS services were restricted to helicopter-based services in use in 2016 and their longitude-latitude coordinates were derived from multiple sources, including the Civil Aviation Authority, the Hawkes Bay Rescue Helicopter Trust and Google Maps. Road network data was obtained from www.geofabrik.de, an online open street map and spatial data provider.

\section{Geospatial access calculations}

The continuous access times were categorised using relatively fine-grained $(<30,30-45,45-60,60-90, \geq 90 \mathrm{~min})$ categories for descriptive purposes and to ensure the use of the 'golden hour' was sensible for the New Zealand population. Timely access was defined as the proportion of the population or land area from which an injured person was theoretically able to reach an advanced-level hospital within a given time of $60 \mathrm{~min}$ via air and road EMS. Access was calculated using established geospatial methods. ${ }^{717}$ The time required for the nearest air or road

Table 1 Categories of advanced levels of hospital care in New Zealand

\begin{tabular}{lll}
\hline Level of hospital care & Services provided & Hospital locations \\
\hline Level 1 & $\begin{array}{l}\text { Advanced trauma and medical services based around comprehensive } \\
\text { intensive care units with specialist staff. }\end{array}$ & Main population areas \\
Level 2 & $\begin{array}{l}\text { Capable of initial resuscitation and management. If specific intensive } \\
\text { care not available then major trauma and severely ill patients }\end{array}$ & $\begin{array}{l}\text { Regional and rural provincial } \\
\text { transferred to level 1 advanced care. }\end{array}$ \\
& $\begin{array}{l}\text { Run by non-specialist staff, capable of initial resuscitation. Major } \\
\text { trauma and severely ill patients transferred to level 1 advanced care. }\end{array}$ & Small rural areas \\
\hline
\end{tabular}


EMS to reach the geometric centroid of a meshblock, stabilise a patient and then directly travel to the nearest advanced-level receiving hospital was calculated for every meshblock.

Road network travel times included the following assumption obtained from St Johns: immediate response and average road ambulance time at scene $14 \mathrm{~min}$ and 49 s. Road ambulance drive times were calculated using the Open Source Routing Machine (OSRM available at www.project-osrm.org). A detailed description on how spatial data are modelled in OSRM is available elsewhere.$^{18}$ All road calculations assumed ambulances drove at legal road speed, did not stop at intersections, driving conditions were ideal (ie, no congestion) and ambulances drove the shortest route.

Air EMS travel times assumed helicopters flew in straight lines at an operational speed of $182 \mathrm{~km} /$ hour, response time (preparation for lift-off) of $8 \mathrm{~min}$, on scene time of $27 \mathrm{~min}$ and $28 \mathrm{~s}$, plus $32 \mathrm{~s}$ to find a safe pick-up landing site. ${ }^{19}$ Air EMS flight times were calculated using custom written Python code running inside QGIS software.

\section{Sociodemographic variables}

Sociodemographic data on age, sex and ethnicity was obtained by summing the subtotal of each characteristic across all meshblocks, thus estimating the population of New Zealand at the time of the 2013 census. Age was grouped into five categories: 0-14, 15-29, 30-44, 45-64 and $\geq 65$ years. The five categories of ethnicity used were: Māori (indigenous population), Pacific, Asian, New Zealand European, MELAA (Middle Eastern, Latin American, African) and Other. Census ethnicity data is not prioritised, with individuals able to record multiple ethnicities, therefore all ethnicities reported are counted resulting in totals greater than $100 \%$.

Deprivation was derived using the 2013 New Zealand Deprivation Index (NZDep) which aggregates and ranks communication, income, employment, family support, qualifications, home ownership, living space and transport within each meshblock area. ${ }^{20}$ NZDep deciles were categorised into quintiles, with ' $1-2$ ' representing the least deprived $20 \%$ and ' $9-10$ ' the most deprived $20 \%$ of meshblocks in New Zealand. Population density within each meshblock area was calculated using number of usually resident people divided by meshblock area, grouped into three categories: high $(20$ to $<200000)$, medium (2 to $<20)$ and low $(0$ to $<2)$ persons $/ \mathrm{km}^{2}{ }^{2}$

\section{Statistical analyses}

Our unit of analysis was the 2013 census meshblock. The count of usually resident populations within each census 2013 meshblock was summed across the country to obtain national denominators. The sociodemographic, deprivation and urbanity characteristics of the population unable to access advanced-level care within key times are described using row percentages. Binomial exact 95\% CIs were calculated for row percentages using Stata V.13 SE.
A map of New Zealand was created to provide a visual representation of the areas within New Zealand that cannot access advanced-level hospital care via air and road EMS within $60 \mathrm{~min}$. Where both air and road were viable options, the shorter time by whatever means was chosen.

\section{Patient and public involvement}

This study is a theoretical modelling geospatial analysis with no direct contact with study participants or patients or the public thus they were not involved in: (1) developing the research question and outcome measures; (2) planning the study design; and (3) the recruitment and conduct of the study. A study advisory group included patient and client representatives from SafeKids New Zealand, St Johns New Zealand, Pacifica Injury Prevention Aukilana, Fire and Emergency New Zealand, ACC and National Ambulance Sector Office. Dissemination of the study's findings will include meetings where patients/ public and community groups engage, including the public health and injury prevention and control meetings.

\section{RESULTS}

Access to advanced-level hospital care by air or road EMS takes over $60 \mathrm{~min}$ for the majority ( $84 \%$ ) of New Zealand's land area. While less than $16 \%$ of our land area allows emergency access via road or air $(3.5 \%$ accessible by road only and $15 \%$ by air only) within $60 \mathrm{~min}$, a substantial majority (83\%) of New Zealanders live within those areas that do have timely access to advanced hospital level care within $60 \mathrm{~min}$ (table 2). Close to a quarter (24\%) of New Zealanders are estimated to have access to advanced-level hospital care within $30 \mathrm{~min}$ and two-thirds $(66 \%)$ within $45 \mathrm{~min}, 13 \%$ of the population are estimated to take up to 30 min longer than the 'golden hour' (ie, within $60-90 \mathrm{~min})$, and $4 \%$ are estimated to take greater than $90 \mathrm{~min}$ to access to advanced-level hospital care.

Population differences in timely access were observed by ethnicity and population density. Persons identifying with the Asian ethnic group have the best estimated access to hospital-level care, with only $0.78 \%$ residing in meshblocks greater than 90 min away from levels 1 or 2 hospital care. In contrast, those of Māori ethnicity had the poorest level of access with $4 \%$ living in meshblocks greater than $90 \mathrm{~min}$ away from advanced-level hospital care (difference in proportion $3.22 \%$, 95\% CI $2.19 \%$ to $4.48 \%)$. With respect to population density, access was poorest for people usually residing in lower density areas meshblocks, with $27 \%$ of people usually resident in low density areas vs $2 \%$ of people usually resident in high density areas being greater than $90 \mathrm{~min}$ away from either levels 1 or 2 hospital care (difference in proportion $25 \%$, $95 \%$ CI $22 \%$ to $27 \%$ ).

Timely geographical access to our most advanced level 1 hospital care is poorest for those aged 65 years or greater, those of Māori, New Zealand European and Other ethnic groups, residents with high levels of deprivation, and 
Table 2 Overall population and sub-population access to advanced-level hospital care by road and helicopter EMS within specified time periods

\begin{tabular}{|c|c|c|c|c|c|c|}
\hline \multirow{2}{*}{$\begin{array}{l}\text { Sociodemographic } \\
\text { characteristics }\end{array}$} & \multirow{2}{*}{$\begin{array}{l}\text { Population } \\
\text { frequency }\end{array}$} & \multicolumn{5}{|c|}{$\begin{array}{l}\text { Have access to levels } 1 \text { or } 2 \text { hospital care within each specified } \\
\text { period Minutes (row \%) }\end{array}$} \\
\hline & & Within 30 & $30-45$ & $45-60$ & $60-90$ & $>90$ \\
\hline Total population & 4240791 & 24 & 42 & 17 & 13 & 4 \\
\hline \multicolumn{7}{|l|}{ Age (years) } \\
\hline $0-14$ & 849192 & 22 & 44 & 19 & 13 & 4 \\
\hline $15-29$ & 829182 & 32 & 41 & 15 & 10 & 3 \\
\hline $30-44$ & 812718 & 25 & 44 & 17 & 11 & 4 \\
\hline $45-64$ & 1065417 & 22 & 42 & 19 & 14 & 4 \\
\hline$\geq 65$ & 605793 & 22 & 40 & 18 & 17 & 4 \\
\hline \multicolumn{7}{|l|}{ Sex } \\
\hline Male & 2061636 & 24 & 42 & 17 & 13 & 4 \\
\hline Female & 2176134 & 24 & 42 & 18 & 13 & 4 \\
\hline \multicolumn{7}{|l|}{ Ethnicity* } \\
\hline New Zealand European & 2963721 & 22 & 40 & 19 & 15 & 3 \\
\hline Māori & 595170 & 23 & 36 & 19 & 17 & 4 \\
\hline Pacific & 295458 & 26 & 54 & 15 & 5 & 1 \\
\hline Asian & 470793 & 32 & 57 & 7 & 3 & 1 \\
\hline MELAA & 46905 & 31 & 52 & 9 & 5 & 2 \\
\hline Other & 67398 & 24 & 42 & 17 & 13 & 3 \\
\hline Not stated & 235437 & 24 & 42 & 17 & 13 & 3 \\
\hline \multicolumn{7}{|l|}{ NZ Depreviation Index } \\
\hline 1-2 (least deprived) & 873393 & 14 & 54 & 21 & 7 & 4 \\
\hline $3-4$ & 85626 & 19 & 45 & 19 & 14 & 4 \\
\hline $5-6$ & 837558 & 24 & 39 & 17 & 17 & 4 \\
\hline $7-8$ & 829704 & 32 & 35 & 15 & 16 & 4 \\
\hline 9-10 (most) & 833169 & 32 & 36 & 15 & 13 & 4 \\
\hline \multicolumn{7}{|c|}{ Population density (persons per $\mathrm{km}^{2}$ ) } \\
\hline High $(20-<200000)$ & 3859551 & 26 & 46 & 14 & 58 & 2 \\
\hline Medium $(2-<20)$ & 303696 & 0.3 & 8 & 37 & 45 & 10 \\
\hline Low $(0-<2)$ & 77544 & 0.015 & 1 & 14 & 57 & 27 \\
\hline
\end{tabular}

*Multiple ethnic identities possible in census; therefore, ethnic categories will add up to more than the total population.

EMS, emergency medical services; MELAA, Middle Eastern, Latin American, African; NZ, New Zealand.

those usually residing in the central North Island and lower South Island (table 3). The addition of regional level 2 hospital care significantly expands the catchment from $59.8 \%$ (95\% CI $56.7 \%$ to $62.9 \%$ ) of the population residing in meshblocks with access to advanced-level hospital care (levels 1 and 2) within $60 \mathrm{~min}$ to $84.6 \%$ (95\% CI $82.2 \%$ to $86.8 \%$ ) (table 3 ). In terms of land area access, this equates to $5 \%$ (95\% CI $4 \%$ to $7 \%$ ) of New Zealand's total land area with timely access to level 1 hospital care within $60 \mathrm{~min}$ which increases to $16 \%$ (95\% CI $14 \%$ to $18 \%$ ) with the addition of level 2 hospital care (results not in table).

Sub-population differences exist in timely access observed by age, ethnicity, level of deprivation and population density (table 3 ). The oldest New Zealanders (65+ years old) have the least access to level 1 (50.7\%, $95 \%$ CI $47.6 \%$ to $53.8 \%$ ) and combined level 1 and 2 hospital care $(79.4 \%, 95 \%$ CI $76.8 \%$ to $81.9 \%)$. Less than half of the Māori population $(45.6 \%, 95 \%$ CI $42.5 \%$ to $48.7 \%$ ) reside in geographical areas with timely access to level 1 hospital care, increasing to $78.7 \%$ (95\% CI $76.0 \%$ to $81.2 \%$ ) with the inclusion of level 2 hospital care, but still substantially lower than the Pacific, Asian and MELAA other ethnic groups. Geographical areas with timely theoretical access to hospital care have a high proportion of resident Asian, Pacific and MELAA ethnic groups. The proportion of the population with timely access to advanced-level hospital care declines with increasing 
Table 3 Usually resident population access to different levels of advanced-level hospital care by road and helicopter EMS within specified time periods

\begin{tabular}{|c|c|c|c|c|}
\hline \multirow[b]{2}{*}{ Demographic characteristics } & \multicolumn{2}{|c|}{$\begin{array}{l}\text { Level } 1 \text { hospital access } \\
\text { Within } 60 \mathrm{~min}\end{array}$} & \multicolumn{2}{|c|}{$\begin{array}{l}\text { Levels } 1 \text { and } 2 \text { hospital access } \\
\text { Within } 60 \mathrm{~min}\end{array}$} \\
\hline & Row \% & $95 \% \mathrm{Cl}$ & Row \% & $95 \% \mathrm{Cl}$ \\
\hline TOTAL & 59.8 & 56.7 to 62.9 & 84.6 & 82.2 to 86.8 \\
\hline \multicolumn{5}{|l|}{ Age (years) } \\
\hline $0-14$ & 59.8 & 56.7 to 62.8 & 84.3 & 81.8 to 86.5 \\
\hline $15-29$ & 67.3 & 64.3 to 70.2 & 88.0 & 85.8 to 89.9 \\
\hline $30-44$ & 64.3 & 61.2 to 67.2 & 86.1 & 83.8 to 88.2 \\
\hline $45-64$ & 58.0 & 54.9 to 61.1 & 82.6 & 80.1 to 84.9 \\
\hline $65+$ & 50.7 & 47.6 to 53.8 & 79.4 & 76.8 to 81.9 \\
\hline \multicolumn{5}{|l|}{ Sex } \\
\hline Male & 59.7 & 56.6 to 62.8 & 83.4 & 80.9 to 85.7 \\
\hline Female & 59.9 & 56.8 to 62.9 & 83.9 & 81.4 to 86.1 \\
\hline \multicolumn{5}{|l|}{ Ethnicity } \\
\hline New Zealand European & 55.3 & 52.2 to 58.4 & 81.5 & 78.9 to 83.9 \\
\hline Māori & 45.6 & 42.5 to 48.7 & 78.7 & 76.0 to 81.2 \\
\hline Pacific & 84.4 & 82.0 to 86.6 & 94.7 & 93.1 to 96.0 \\
\hline Asian & 87.4 & 85.1 to 89.4 & 96.1 & 94.7 to 97.2 \\
\hline MELAA & 82.9 & 80.4 to 85.2 & 93.0 & 91.2 to 94.5 \\
\hline Other & 52.7 & 49.6 to 55.8 & 80.8 & 78.2 to 83.2 \\
\hline Not stated & 59.9 & 56.8 to 62.9 & 83.7 & 81.2 to 85.9 \\
\hline \multicolumn{5}{|l|}{ NZ Deprivation Index } \\
\hline 1-2 (least deprived) & 73.4 & 70.5 to 76.1 & 90.0 & 87.9 to 91.8 \\
\hline $3-4$ & 62.0 & 58.9 to 65.0 & 83.0 & 81.5 to 85.3 \\
\hline $5-6$ & 54.9 & 51.8 to 58.0 & 79.9 & 77.3 to 82.3 \\
\hline $7-8$ & 53.9 & 50.8 to 57.0 & 80.5 & 77.9 to 82.9 \\
\hline 9-10 (most deprived) & 55.1 & 51.9 to 58.2 & 83.6 & 81.2 to 85.8 \\
\hline \multicolumn{5}{|c|}{ Population Density (persons per $\mathrm{km}^{2}$ ) } \\
\hline High (20-<200 000) & 64.3 & 61.2 to 67.2 & 88.0 & 85.8 to 89.9 \\
\hline Medium $(2-<20)$ & 17.1 & 14.8 to 19.6 & 45.3 & 42.2 to 48.4 \\
\hline Low $(0-<2)$ & 2.8 & 1.8 to 4.0 & 15.2 & 13.0 to 17.6 \\
\hline
\end{tabular}

EMS, emergency medical services; MELAA, Middle Eastern, Latin American, African; NZ, New Zealand.

levels of deprivation, the exception to this is the most deprived group (NZDep 9-10) for whom access increased slightly from the previous level of NZDep 7-8, reflecting many of highly deprived areas in New Zealand are located in main urban areas close to hospitals. Those residing in meshblock areas with the least deprivation have a substantially better degree of timely access. Regions with low to medium population density have the poorest access to prehospital care, with a paltry $2.8 \%(95 \%$ CI $1.8 \%$ to $4.0 \%$ ) of those usually residing in areas of low population density having access to a level 1 hospital, expanding to $15.2 \%$ (95\% CI $13.0 \%$ to $17.6 \%$ ) with the inclusion of level 2 care. No differences were observed by sex.

Figure 1 provides a visual representation of the catchment of air and road EMS given the placement of advanced-level hospitals and EMS bases. Comparing figure 1A,C provides some illustration of why timely access to usually resident population is high, but land area access is low. Timely access is clustered around the main highly populated areas with a population density in the range 20 to $<200000$ people $/ \mathrm{km}$. Air EMS services improve the level of timely access to advanced hospital level care above and beyond road EMS services in all areas except two in the lower South Island. However, much of the coverage is 'doubled-up' as the combined $\mathrm{road} / \mathrm{air}$ catchment closely follows the road only catchment. Many areas of moderate population density are not adequately covered by EMS, and the most isolated pockets of non-coverage are in areas with low population density. 


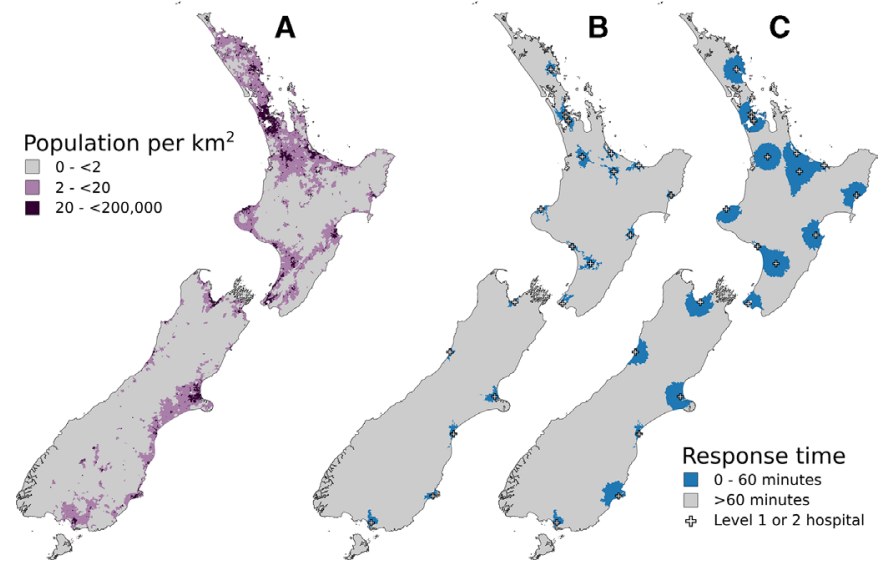

Figure 1 Access to advanced-level hospital care in New Zealand within 60 min. (A) Population density. (B) Road emergency medical services (EMS) coverage. (C) Road or air EMS coverage.

\section{DISCUSSION}

This research examined the proportion of the usually resident population with theoretical timely access to advanced-level hospital care by air and road EMS in New Zealand and identified important disparities in access to these prehospital services. Not surprisingly, timely access is clustered around areas of high population density based on where New Zealand's population usually resides. Applying the concept of the 'golden hour', we estimate $16 \%$ of the New Zealand population does not have timely theoretical access to advanced-level hospital care via road or air EMS. This equates to around 700000 New Zealanders without timely access; particular disparities in theoretical timely access were observed for Māori, European New Zealanders, older New Zealanders and those residing in the lower South Island. Regionally based level 2 hospital services are vital for rural communities to have timely access to care, particularly the Central North Island region where we estimate there is no timely access to the most advanced level 1 hospital care within $60 \mathrm{~min}$.

Previous international studies examining timely theoretical access to care following time-critical acute medical and trauma events have mainly focused on access to trauma services. Prehospital access to advanced trauma care in New Zealand is similar to that observed in the USA and Canada. ${ }^{7910}$ Timely access to advanced trauma care via road or air EMS was available to $88 \%$ of all US residents, while $77 \%$ of Canadian residents have access via road ambulances. ${ }^{910}$ Access to critical care services in Scotland is more comprehensive than New Zealand with $94 \%$ of the Scottish population within a $45 \mathrm{~min}$ ambulance drive to definitive hospital-level care covering $47 \%$ of the Scottish landmass. ${ }^{21}$ Unsurprisingly, the catchment of EMS and advanced-level hospital care aligns closely to population density in New Zealand, as also demonstrated in the USA, Canada and Scotland. Advanced-level hospital services are located to serve a large population base making the most efficient use of the resource and maintaining high levels of medical skill and management.
This focus on areas of high population density, however, means that areas of disparities are generated on the basis of population density and dispersion. Areas of low to medium population density that have poorer EMS coverage typically have disproportionately greater indigenous Māori and ageing New Zealand European populations, high-risk economic activity (such as farming and forestry) and remote areas of recreational and tourist activity.

Our study found important socio-demographic disparities in timely theoretical access for indigenous Māori, New Zealand Europeans, older New Zealanders and those residing in the southern part of the South Island, reflecting the geographical spread of New Zealand's population. While most Māori live in urban areas, Māori make up a higher proportion of the population residing in highly rural and remote areas, by contrast almost all Pacific and the majority of Asian people in New Zealand live in urban areas. ${ }^{22}$ Older New Zealanders, who are typically New Zealand European, contribute to a high proportion of those who live in rural areas with low to moderate urban influence, explaining the longer theoretical access times observed for this group. Regions with low to moderate population density stood out as being significantly underserved when compared with areas of high population density. Similar disparities in access to advanced-level hospital care have been reported internationally for those residing in rural areas or very remote areas, and for ethnic minorities. ${ }^{7-11} 21$ Our study provides further evidence of disparities in timely theoretical access to healthcare for Māori and for rural communities, and the unequal distribution of healthcare resources across New Zealand providing a further pathway for health disparities for Māori and rural populations: difficulties in achieved access to healthcare for these sub-groups are already well documented. ${ }^{2324}$ Our results add further support for EMS and advanced-level hospital services to be optimally configured to address both the geospatial and socio-demographic challenges of timely access relative to population density in New Zealand.

The optimal placement or enhancement of EMS retrieval and transfer services and advanced healthcare facilities in New Zealand should be examined further. One of the guiding principles for New Zealand's health system is that all New Zealanders have 'timely and equitable access' to a 'comprehensive range of health and disability services'. ${ }^{25}$ Our study found concerning geospatial disparities in timely geographical access for communities with low to moderate population density which tend to be rural and remote communities with disproportionately more Māori and older New Zealand Europeans. Furthermore, areas with few permanent residents are popular destinations for international and residential tourists travelling to, and engaging in, outdoor tourist pursuits in remote back country and alpine areas. We have identified the need for the combined reach of EMS services and advanced-level hospital care to consider 
coverage of socio-demographic and geospatial aspects beyond just high density residential population bases.

Understanding the geospatial distribution of EMS services and timely access into advanced-level hospital care is the first step in identifying opportunities for improved care and planning of future services and health policy in the prehospital setting of an advanced trauma and medical system. It is unknown if this current level of under-service results in higher rates of mortality across New Zealand, and future analyses from this research team will examine this question further with regard to injury. ${ }^{17}$ Geographical areas with limited prehospital air and road EMS may be adequately served by other groups of appropriated trained first responders, such as general practitioners, police and fire services, where they sit outside of existing EMS catchment areas. Future research should examine the location of other first responders and the expansion of coverage of EMS these groups potentially provide. Other approaches to extending access to EMS and advanced-level hospital care include increasing, upgrading or integrating the roles of regional level 3 hospitals in the provision of emergency care; expanding helicopter provision, repositioning existing road or air EMS depots, or providing novel methods such as delivery of advanced care to critically injured patients or telemedicine interventions. $^{710}$

This study has a number of limitations. The choice of geospatial software and assumptions made are likely to impact the theoretical access estimates presented; we have consciously chosen conservative assumptions and settings meaning the proportion without timely access is likely to be underestimated. This analysis also assumes simultaneous dispatch by road and air EMS services, however the operational reality is that air response can take longer when emergency first responders are required to assess severity of an acute event prior to helicopter dispatch. It also assumes the availability of suitable helicopter landing sites at the meshblock centroid which may not be available. The benefits of the extended air ambulance coverage identified in our analysis would therefore only be achieved if air ambulance services were dispatched without delay and if suitable helicopter landing sites were available. Early/simultaneous activation models have shown clear benefits for prevention of needless deaths. ${ }^{26}{ }^{27}$ A further limitation is that the EMS scenario theoretically modelled in our analysis fits a 'scoop \& run' strategy to prehospital care, and does not consider other possible regional or local prehospital and EMS strategies, such as a differentiated strategy, or rapid delivery of specialist medical expertise to the patient. ${ }^{28}$ Recent empirical evidence suggests that a prehospital strategy should differentiate by injury type, with the worst case scenario injuries involving penetrating haemodynamically unstable injury or neurological trauma needing to reach advanced care as quickly as possible to have the best chance of survival. ${ }^{29}$ The use of centroids may have led to geographical selection bias when a meshblock sits on edge of the time boundary, yet the centroid sits outside this boundary and is not selected. The potential for geographical selection bias is thought to be balanced out by the inclusion of similar sized meshblock's in close proximity with partial coverage over a time boundary where the centroid sits inside the boundary. Manual review of the 1044 large, remote meshblocks where the centroid was $10 \mathrm{~km}$, or greater, from the nearest road revealed no part of these area units was reachable by air or road EMS within the time-limits examined.

Our study expands on previous geospatial examinations of access in New Zealand ${ }^{30}$ and internationally ${ }^{7} 1021$ with the use of actual location of road-based and air-based EMS services, with comparison to usually resident population densities, to assess the actual level of EMS coverage and response providing valuable geospatial analyses to inform future planning and expansion of prehospital EMS in New Zealand. As New Zealand is an island nation with a universal nationally funded healthcare system, we are able to consider the closest hospital care without being restricted by state, provincial or national funding boundaries, unlike some international studies.

\section{CONCLUSION}

Timely access to advanced-level hospital care is important to increase survival from time-critical acute injury and medical events. Our study highlights the need for planning of EMS coverage in New Zealand, especially for areas of moderate to low population density, in order to address inequities with regard to timely access to advanced-level hospital care. The geographical configuration of EMS and healthcare systems is important for optimising accessibility to services and promoting the efficient use of scarce resources in a geographically challenging island nation. Future analyses will examine if disparities in timely access to advanced-level hospital care translates into higher rates of mortality due to time-critical injuries in New Zealand and identify areas requiring improved access to EMS and advanced-level hospital care relative to the burden of prehospital fatal injury. ${ }^{17}$

\section{Author affiliations}

${ }^{1}$ Department of Preventive and Social Medicine, Division of Health Sciences, University of Otago, Dunedin, New Zealand

${ }^{2}$ Section of Epidemiology and Biostatistics, The University of Auckland, Auckland, New Zealand

${ }^{3}$ Te Kupenga Hauora Māori, The University of Auckland, Auckland, New Zealand ${ }^{4}$ Department of Paramedicine, Auckland University of Technology, Auckland, New Zealand

${ }^{5}$ St Johns, Auckland, New Zealand

${ }^{6}$ Department of Surgery, The University of Auckland, Auckland, New Zealand

${ }^{7}$ Trauma and Vascular Surgery, Auckland City Hospital, Auckland, New Zealand

${ }^{8}$ Department of Epidemiology, Columbia University, New York city, New York, USA

Acknowledgements The authors would like to thank lan Wilmot (Wilmot Consulting), and staff of the St John, Wellington Free Ambulance and Ministry of Health for supplying data integral to our geospatial analyses.

Contributors RL contributed to the study design, conducted the literature review, provided oversight of the spatial analysis and was primarily responsible for the article preparation. BdG was primarily responsible for acquiring data and the spatial analysis, as well as contributing to the interpretation of data and preparation of the 
paper. GD contributed to the study design, provided advice on the spatial analysis methods, overseeing the spatial analysis and contributed to the interpretation of data and preparation of the paper. BK contributed to the study design, provided oversight of the project, interpretation of results and article preparation. CB contributed to the study design, provided advice on the spatial analysis methods and contributed to the direction of the analysis and interpretation of data and preparation of the paper. PR, SA, IC and BD contributed to the study design and to the interpretation of data and preparation of the paper.

Funding This research was funded by the Health Research Council of New Zealand project grant (HRC Grant 15/186).

Map disclaimer The depiction of boundaries on the map(s) in this article do not imply the expression of any opinion whatsoever on the part of BMJ (or any member of its group) concerning the legal status of any country, territory, jurisdiction or area or of its authorities. The map(s) are provided without any warranty of any kind, either express or implied.

Competing interests None declared.

Patient consent for publication Not required.

Ethics approval Ethical approval for the broader programme of research, of which this specific study is a part of, was obtained from the University of Auckland Human Participants Ethics Committee (ref 016179), National Coronial Information System (ref NZ007) and University of Otago Health and Disability Ethics Committee (ref 0TA/90/02/008/AM05).

Provenance and peer review Not commissioned; externally peer reviewed.

Data availability statement Data are available upon reasonable request.

Open access This is an open access article distributed in accordance with the Creative Commons Attribution Non Commercial (CC BY-NC 4.0) license, which permits others to distribute, remix, adapt, build upon this work non-commercially, and license their derivative works on different terms, provided the original work is properly cited, appropriate credit is given, any changes made indicated, and the use is non-commercial. See: http://creativecommons.org/licenses/by-nc/4.0/.

\section{REFERENCES}

1. Sasser S, Varghese M, Kellermann A, et al. Prehospital trauma care systems. Geneva: World Health Organization, 2005.

2. Waller AE, Baker SP, Szocka A. Childhood injury deaths: national analysis and geographic variations. Am J Public Health 1989;79:310-5.

3. Centers for Disease Control and Prevention. QuickStats: percentage of injury deaths for which death was pronounced outside of a hospital, by leading mechanism of injury deaths: United States, 2005. MMWR 2008;57.

4. RACS NZ Trauma Committee. Guidelines for a structured approach to the provision of optimal trauma care. Wellington Ministry of Health; 1994.

5. Boersma E, Maas ACP, Deckers JW, et al. Early thrombolytic treatment in acute myocardial infarction: reappraisal of the golden hour. The Lancet 1996;348:771-5

6. Spiotta AM, Vargas J, Turner R, et al. The golden hour of stroke intervention: effect of thrombectomy procedural time in acute ischemic stroke on outcome. J Neurointerv Surg 2014;6:511-6.

7. Branas CC, MacKenzie EJ, Williams JC, et al. Access to trauma centers in the United States. JAMA 2005;293:2626-33.
8. TraumaMaps.org. University of Pennsylvania cartographic modeling laboratory; 2006.

9. Carr BG, Bowman AJ, Wolff CS, et al. Disparities in access to trauma care in the United States: a population-based analysis. Injury 2017;48:332-8

10. Hameed SM, Schuurman N, Razek T, et al. Access to trauma systems in Canada. J Trauma 2010;69:1350-61.

11. Hsia R, Shen Y-C. Possible geographical barriers to trauma center access for vulnerable patients in the United States: an analysis of urban and rural communities. Arch Surg 2011;146:46-52.

12. National Health Service, The NHS Atlas of Variation in Healthcare. Reducing unwarranted variation to increase value and improve quality. London National Health Service; 2010.

13. Statistics New Zealand. population, 2018. Available: http://archive. stats.govt.nz/browse for stats/government finance/central government/nz-in-the-oecd/population.aspx [Accessed April 2018]

14. Falconer JA. Preventability of pre-hospital trauma deaths in southern New Zealand. N Z Med J 2010;123:11-9.

15. Grey $C$, Jackson $R$, Schmidt $M$, et al. One in four major ischaemic heart disease events are fatal and $60 \%$ are pre-hospital deaths: a national data-linkage study (ANZACS-QI 8). Euro Heart $J$ 2017;38:172-80.

16. Statistics New Zealand. Geographic boundary files Wellington: statistics New Zealand, 2015. Available: www.stats.govt.nzll browse-for-stats $\backslash M a p s-a n d-g e o g r a p h y \backslash \backslash G e o g r a p h i c-a r e a s \backslash \backslash d i g i t a l-$ boundary-files.aspx [Accessed February 2017].

17. Lilley R, Kool B, Davie G, et al. Preventable injury deaths: identifying opportunities to improve timeliness and reach of emergency healthcare services in New Zealand. Inj Prev 2018;24.

18. Luxen D, Luxen D, Vetter C. Real-Time routing with OpenStreetMap data. GIS'11 Proceedings of the 19th ACM SIGSPATIAL International Conference on Advances in Geographic Information Systems, Chicago, Illinois, 2011.

19. Gordon K, Swain A, Thirkell C, et al. The Wellington life flight helicopter emergency medical service (HemS): a retrospective audit against new Ministry of health criteria. N Z Med J 2014;127:30-42.

20. Atkinson J, Salmond C, Crampton P. NZDep2013 index of deprivation. Wellington: University of Otago, 2014.

21. Emerson P, Dodds N, Green DR, et al. Geographical access to critical care services in Scotland. J Intensive Care Soc 2018;19:6-14.

22. Statistics New Zealand. New Zealand: an urban/rural profile. Wellington Statistics New Zealand; 2004.

23. Ellison-Loschmann L, Pearce N. Improving access to health care among New Zealand's Maori population. Am J Public Health 2006;96:612-7.

24. Nixon G, Samaranayaka A, de Graaf B, et al. Geographic disparities in the utilisation of computed tomography scanning services in southern New Zealand. Health Policy 2014;118:222-8.

25. Ministry of Health. New Zealand health strategy future direction Wellington Ministry of Health; 2016.

26. Diaz MA, Hendey GW, Bivins HG. When is the helicopter faster? A comparison of helicopter and ground ambulance transport times. $J$ Trauma 2005;58:148-53.

27. Hankins DG. Air medical transport of trauma patients. Prehosp Emerg Care 2006;10:324-7.

28. Smith RM, Conn AKT. Prehospital care - scoop and run or stay and play? Injury 2009;40 Suppl 4:S23-S26.

29. Harmsen AMK, Giannakopoulos GF, Moerbeek PR, et al. The influence of prehospital time on trauma patients outcome: a systematic review. Injury 2015;46:602-9.

30. Brabyn L, Skelly C. Modeling population access to new Zealand public hospitals. Int J Health Geogr 2002;1:3. 